\title{
The effects of whole almond snack consumption on fasting blood lipids and insulin sensitivity: a randomised controlled trial in adults
}

\author{
V. Dikariyanto ${ }^{1}$, S. E. E. Berry ${ }^{1}$, L. Smith $^{1}$, L. Francis ${ }^{1}$, M. Robertson ${ }^{1}$, E. Kusaslan ${ }^{1}$, \\ M. O’Callaghan-Latham ${ }^{1}$, P. R. Ellis ${ }^{2}$ and W. L. Hall ${ }^{1}$ \\ ${ }^{1}$ Diet and Cardiometabolic Health Research Group, Department of Nutritional Sciences, Faculty of Life Science and \\ Medicine, King's College London, London SE1 9NH, UK and \\ ${ }^{2}$ Biopolymers Group, Faculty of Life Science and Medicine, King's College London, London SE1 9NH
}

Sub-optimal dietary patterns associated with many snack foods, such as consumption of high-fat, high-refined carbohydrate foods that are low in fibre and nutrients, present an easily identifiable target to improve cardio-metabolic health. The potential health benefits of snacking on almonds may be the result of displacement of other snacks high in refined carbohydrates, the addition of nutrients and non-nutritive bioactive compounds into the diet, and/or low lipid bioaccessibility ${ }^{(1)}$. A recent meta-analysis of prospective epidemiological studies found that increased nut consumption is associated with lower CVD risk ${ }^{(2)}$ and another meta-analysis of randomised controlled trials reported that almonds reduced LDL-cholesterol whilst maintaining HDL-cholesterol ${ }^{(3)}$. The impact of almond snack consumption on insulin sensitivity and related circulating markers of metabolic health remains unclear.

A 6-week randomised, controlled, parallel trial (ClinicalTrials.gov NCT02907684) in regular snack consumers at moderate risk of CVD (aged 30-70 y) was conducted aiming to investigate whether replacing habitual snacks (20\% estimated daily energy requirements) with whole roasted almonds had any impact on blood lipid levels and insulin sensitivity. Control snacks (sweet and savoury mini-muffins) were developed to simulate the average UK snack nutrient profile, which was calculated from snack foods identified in the UK NDNS database ${ }^{(4)}$. One hundred and nine volunteers were randomised to treatment ( 77 females, 32 males) with minimisation for age, sex, ethnicity, cardiometabolic score and whether or not they consented to undertake MRI scanning; 106 completed the study. Fasting blood samples were taken at baseline and the end of week-6. Total plasma cholesterol, triglycerides, LDL-cholesterol, HDL-cholesterol, glucose, insulin and markers related to insulin sensitivity, including adiponectin, leptin, resistin and fetuin-A were analysed. HOMA-IR was calculated as an index of insulin resistance. Statistical analysis was performed on changes from baseline using ANCOVA adjusted for age, sex, baseline body mass index and baseline variable value.

Plasma LDL-cholesterol concentrations were $0.25 \mathrm{mmol} / \mathrm{L}(95 \% \mathrm{CI}-0.47,-0.03)$ lower following almonds relative to control, but there were no statistically significant treatment differences for HDL-cholesterol, total cholesterol, triglycerides, insulin, glucose, nor HOMA-IR. Furthermore, there were no significant differences between treatment groups for serum adiponectin, leptin, resistin and fetuin-A. Estimated marginal means for changes from baseline following both treatments are presented in Table 1.

Table 1. Estimated marginal mean changes from baseline following 6-week

snack replacement intervention

\begin{tabular}{|c|c|c|c|}
\hline \multirow[b]{2}{*}{ Variable } & \multicolumn{2}{|c|}{$\begin{array}{l}\text { Mean of change } \\
\text { values }(95 \% \mathrm{CI})\end{array}$} & \multirow[b]{2}{*}{$\begin{array}{c}P \\
\text { value }\end{array}$} \\
\hline & $\begin{array}{c}\text { Control group, } \\
n=49\end{array}$ & $\begin{array}{l}\text { Almond group, } \\
n=53\end{array}$ & \\
\hline Total cholesterol (mmol/L) & $-0.00(-0.19,0.19)$ & $-0.22(-0.41,-0.04)$ & 0.096 \\
\hline Triglycerides $(\mathrm{mmol} / \mathrm{L})$ & $-0.08(-0.19,0.03)$ & $-0.03(-0.13,0.07)$ & 0.464 \\
\hline LDL-cholesterol (mmol/L) & $0.12(-0.04,0.29)$ & $-0.13(-0.28,0.03)$ & $0.030 *$ \\
\hline HDL-cholesterol (mmol/L) & $0.02(-0.07,0.10)$ & $-0.06(-0.14,0.01)$ & 0.170 \\
\hline Insulin (mlU/L) & $1.46(-0.02,2.93)$ & $0.62(-0.76,2.01)$ & 0.416 \\
\hline Glucose (mmol/L) & $0.05(-0.13,0.22)$ & $0.06(-0.10,0.22)$ & 0.928 \\
\hline HOMA-IR & $0.42(0.01,0.83)$ & $0.21(-0.18,0.59)$ & 0.450 \\
\hline Adiponectin (mg/L) & $-0.26(-0.94,0.41)$ & $-0.16(-0.80,0.48)$ & 0.831 \\
\hline Leptin $(\mu \mathrm{g} / \mathrm{L})$ & $-0.85(-2.87,1.17)$ & $0.25(-1.60,2.10)$ & 0.426 \\
\hline Resistin $(\mu \mathrm{g} / \mathrm{L})$ & $0.09(-0.27,0.45)$ & $-0.00(-0.34,0.34)$ & 0.707 \\
\hline Fetuin-A (mg/L) & $15.18(-23.18,53.54)$ & $4.33(-31.71,40.36)$ & 0.684 \\
\hline
\end{tabular}

ANCOVA was used, adjusted for age, sex, baseline BMI and baseline variable value. ${ }^{*} \mathrm{p}<0.05$

indicates statistical significance.

Results are consistent with previous literature in showing that habitual consumption of whole almonds results in reduced LDL-cholesterol concentrations, suggesting that a simple snack swap could be beneficial for lipid profiles even if other meals remain unmodified. However, no differences were observed for other lipid markers, and there was no impact of a snack replacement intervention on a range of insulin sensitivity markers. Future research should investigate whether replacing nutritionally poor quality snacks with almonds has an impact on insulin sensitivity in study populations with pre-diabetes.

1. Ros E (2010) Nutrients 2, 652-682

2. Musa-Veloso K, Paulionis L et al. (2016) J Nutr Sci 5, e34.

3. Mayhew AJ, de Souza RJ et al. (2016) Br J Nutr 115(2), 212-225.

4. National Diet and Nutrition Survey (2008/2009-2013/14). Sep 2016. Public Health England/UK Food Standards Agency. 